\title{
Preparation for horizontal or vertical dimensions affects the right-left prevalence effect
}

\author{
AKIo Nishimura AND Kazuhiko Yokosawa \\ University of Tokyo, Tokyo, Japan
}

\begin{abstract}
When stimulus and response simultaneously vary in both horizontal and vertical dimensions, the stimulusresponse compatibility effect is often larger for the horizontal dimension. We investigated the role of preparation for each dimension in this right-left prevalence. In Experiment 1, tasks based on horizontal and vertical dimensions were mixed in random order, and the relevant dimension in each trial was cued with a variable cue-target stimulus onset asynchrony (SOA). A right-left prevalence effect was observed only when participants prepared for the upcoming task. Experiment 2 replicated the absence of the prevalence effect for the simultaneous presentation of cue and target using a fixed SOA of 0 msec. In Experiment 3, the right-left prevalence emerged with a 0 -msec SOA when participants prepared for each dimension based on its frequency. These results suggest that participants' internal set can be greater for the horizontal dimension, leading to the right-left prevalence effect.
\end{abstract}

With dimensionally overlapped stimulus and response sets, responses are faster and more accurate when the stimulus and the response correspond than when they do not (Kornblum, Hasbroucq, \& Osman, 1990). This is called the stimulus-response $(S-R)$ compatibility effect. The S-R compatibility effect has been widely investigated for spatial S-R correspondences (for a review, see Proctor \& Reeve, 1990). In a typical spatial S-R compatibility task, both the stimuli and the responses vary horizontally. Participants are required to make a right or a left response to the stimulus position. Responses are faster and more accurate when the stimuli are assigned to ipsilateral responses (i.e., right response to right stimulus, and left response to left stimulus) than when they are assigned to contralateral responses (i.e., right response to left stimulus, and left response to right stimulus; see, e.g., Brebner, 1973; Shaffer, 1965). The S-R compatibility effect emerges even when the stimulus position is irrelevant to the task (e.g., Simon \& Craft, 1972), known as the Simon effect (for reviews, see Lu \& Proctor, 1995; Simon, 1990). According to the coding explanation of the S-R compatibility effect (e.g., Umiltà \& Nicoletti, 1990; Wallace, 1971), a spatial code that specifies the stimulus position is formed when the stimulus is presented, and is compared with the spatial code that specifies the response position. When the stimulus and response codes are identical, the response selection is faster than when they are not.

The S-R compatibility effect also emerges for the vertical dimension, and the size of the vertical compatibility effect is comparable to the size of the horizontal compatibility effect (Nicoletti \& Umiltà, 1984; Vu, Proctor, \& Pick, 2000). However, when S-R compatibility varies both in the horizontal and the vertical dimensions simultaneously, the compatibility effect is often larger for the horizontal dimension than for the vertical dimension (right-left prevalence; Nicoletti \& Umiltà, 1984; for a review, see Rubichi, Vu, Nicoletti, \& Proctor, 2006). Umiltà and Nicoletti (1990) concluded that people may preferentially allocate attention to the horizontal dimension rather than the vertical dimension, making the horizontal dimension salient. This saliency favors the spatial coding of the horizontal dimension when both horizontal and vertical spatial information are available.

Mainly situational factors of the stimulus or response influencing the right-left prevalence effect have been investigated, such as unimanual versus bimanual responses (Hommel, 1996; Vu \& Proctor, 2001); ipsilateral versus contralateral hand and foot (Vu \& Proctor, 2001, 2002; see also Nicoletti \& Umiltà, 1985); crossed versus uncrossed hands (Vu \& Proctor, 2001, 2002); the distance between the responding hands ( $\mathrm{Vu}$ et al., 2000); stimulus modality (Nicoletti, Umiltà, Tressoldi, \& Marzi, 1988); and the distance between the stimuli along horizontal and vertical dimensions (Vu \& Proctor, 2002) (see also Rubichi et al., 2006). The findings of these studies are consistent with the salient-features coding account proposed by $\mathrm{Vu}$ and Proctor (2001, 2002; Vu et al., 2000). They proposed that right-left prevalence occurs because using the right and left effectors makes the right-left distinction more salient than the top-bottom distinction and that the prevalence effect (i.e., a larger S-R compatibility effect) emerges along the more salient dimension determined by the task structure.

However, internal factors also seem to affect the rightleft prevalence. Hommel (1996, Experiment 1) found an advantage for horizontal compatibility effect relative to

A. Nishimura, akio@L.u-tokyo.ac.jp 
vertical compatibility effect when participants were instructed to choose responses exclusively on the basis of the horizontal dimension. A similar advantage of vertical compatibility effect was found with instruction to choose responses based exclusively on the vertical dimension. Thus, he did not find a "strong" right-left prevalence in which the horizontal compatibility effect was larger than the vertical compatibility effect in spite of the vertical instruction. However, he did find a "weak" right-left prevalence effect in which the advantage of the horizontal compatibility effect for the horizontal instruction was larger than the advantage of the vertical compatibility effect for the vertical instruction. ${ }^{1}$ Hommel (1996) attributed the right-left prevalence reported by Nicoletti and Umiltà (1984, 1985; Nicoletti et al., 1988) to their participants' not following the vertical instruction but instead using the horizontal code to determine the response because of the faster formation of the horizontal code than the vertical code due to the use of right and left effectors (see also Rubichi, Nicoletti, Pelosi, \& Umiltà, 2004, for the close relationship between the speed of code formation and right-left prevalence). Vu et al. (2000) replicated the effect of instruction on the right-left prevalence effect.

Thus, instructions that influence the preparation state of the participants for each dimension have an effect on right-left prevalence (Hommel, 1996; Vu et al., 2000). The absence of right-left prevalence in a two-dimensional Simon task with bimanual keypress responses (Proctor, Vu, \& Nicoletti, 2003; Vu, Pellicano, \& Proctor, 2005), in which the stimulus position was irrelevant to the task, also indicates the importance of participants' intentional preparation for each dimension. ${ }^{2}$ Right-left prevalence may emerge because preparation for the horizontal dimension is stronger than for the vertical dimension due to the relative salience of the horizontal dimension. However, most right-left prevalence studies, except those using a Simon task, varied the conditions between blocks (such as both compatible, horizontally compatible, vertically compatible, and neither compatible) and between participants (such as horizontal and vertical instruction groups). In these conditions, participants could fully prepare for the task in a block before the target in each trial was presented. Moreover, some of the participants might have ignored the task instructions based on the vertical dimension in the previous studies using between-block manipulations of conditions, as was suggested by Hommel (1996).

Some recent studies have used within-block manipulations of conditions. For example, Meiran and his colleagues conducted a series of task-switching studies with S-R sets similar to those used in the right-left prevalence paradigm (Meiran, 1996, 2000a, 2000b, 2005; Meiran \& Chorev, 2005; Meiran, Chorev, \& Sapir, 2000). In Meiran's paradigm (see Figure 1, but note that Figure 1 depicts the experimental setups of our study and is different from Meiran's in some aspects), the participants always responded by using a key that spatially corresponded to the stimuli along the task-relevant dimension. For example, when participants responded using an up-left/down-right key arrangement in the horizontal task, as is depicted in

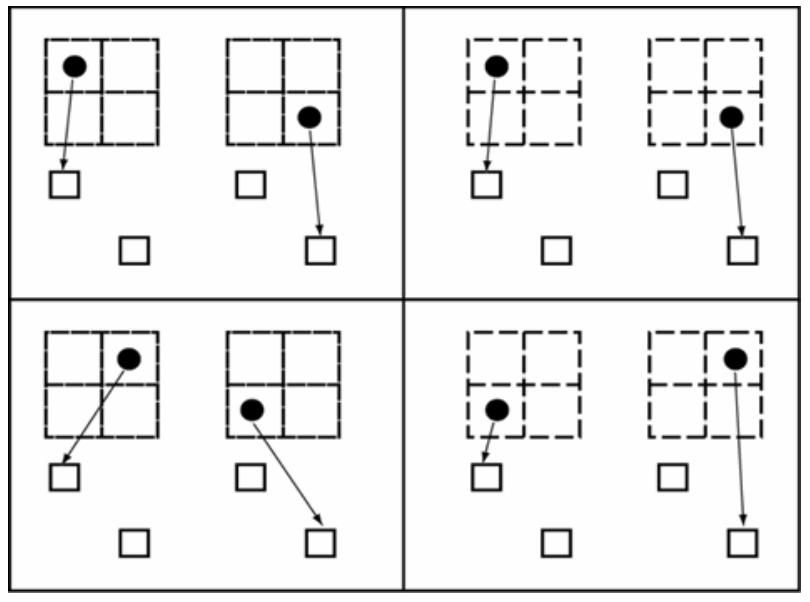

Figure 1. Illustration of the compatibility conditions (compatible in upper panels; incompatible in lower panels) for the vertical task (left panel) and for the horizontal task (right panel), with upleft/down-right key configuration. Stimuli are depicted by filled circles, response keys are depicted by squares, and red and green grids are depicted by two types of dotted lines.

the right panel of Figure 1, the S-R spatial relationship was always compatible along the task-relevant horizontal dimension. However, the task-irrelevant vertical relationship was compatible when the target appeared in the up-left or down-right position (upper panel) but was incompatible when it appeared in the up-right or down-left position (lower panel). Thus, S-R compatibility varied only for the task-irrelevant dimension. Right-left prevalence has been discussed mainly in terms of the overall relevant compatibility effect across the conditions, and of the compatibility effect when the irrelevant dimension was incompatible (see Vu et al., 2000). However, the compatibility effect corresponding to Meiran's paradigm (i.e., the irrelevant S-R compatibility effect when the relevant dimension was always compatible) also seemed to show a right-left prevalence effect in previous studies. For example, Vu et al. (2000, see their Table 1; see also Vu \& Proctor, 2002, Appendix A, for similar results) obtained a larger horizontal compatibility effect (56 msec; defined as the difference between the both compatible and the vertically compatible conditions with vertical instructions, corresponding to the upper left and lower left panels of our Figure 1, respectively) than a vertical compatibility effect $(-2 \mathrm{msec}$; defined as the difference between the both compatible and the horizontal compatible conditions with horizontal instructions, corresponding to the upper right and lower right panels of our Figure 1, respectively). This showed a right-left prevalence effect with irrelevant dimensions when the relevant dimension was compatible.

Meiran (1996) found no difference between the horizontal and the vertical tasks, and no significant effects including the interaction between task and compatibility. Because of these null effects, Meiran rarely included the task as a factor in the analyses in subsequent studies. However, Meiran (2005) recently obtained a larger horizontal compatibility effect with a vertical task than a vertical 
compatibility effect with a horizontal task, and this rightleft prevalence effect was not affected by the interval between the cue onset and the target onset (i.e., the stimulus onset asynchrony, or SOA). Because the SOAs used in Meiran (2005) were limited (200 and 1,200 msec), the difference between vertical and horizontal compatibility effects and the influence of preparation time in this paradigm is unclear.

Recently, Proctor, Koch, and Vu (2006) conducted a right-left prevalence study using a variant of Meiran's paradigm. In Experiments 1 and 2, the stimuli varied only along the incongruent diagonal. In Experiment 3, the stimuli appeared in one of the quadrants, and thus the experimental situation was more similar to that used in Meiran (2005). Proctor et al. (2006) reported a right-left prevalence unaffected by the SOA (100 or $900 \mathrm{msec})$. Thus, they replicated Meiran (2005), and concluded that the task structure determines the right-left prevalence effect and preparation for each dimension does not alter the prevalence effect. However, they used an overall reaction time (RT) in each condition, not the compatibility effect, as an indicator of right-left prevalence. The right-left prevalence effect has been defined as a larger horizontal compatibility effect relative to a vertical compatibility effect. So it may not be strictly equivalent to regard the difference of RTs in horizontally instructed and vertically instructed conditions as evidence for a right-left prevalence effect. In fact, Proctor et al. (2006) included compatibility as a factor for analysis and found an interaction between task and compatibility, indicating a right-left prevalence effect according to our definition. Although the three-way interaction was not significant, the compatibility effect based on our definition was numerically smaller for a short SOA than for a long SOA, indicating a role of preparation in the right-left prevalence effect. The numerical increase of the right-left prevalence effect as a function of SOA in Proctor et al. (2006) suggests very rapid preparation for each dimension. However, both Meiran (2005) and Proctor et al. (2006) included only partial and full preparation conditions (i.e., short and long SOAs) and did not investigate the condition with no preparation time for each dimension. Thus, although the importance of an internal state for right-left prevalence has been indicated (Hommel, 1996; Proctor et al., 2003; Vu et al., 2000, 2005), the role of preparation for each dimension in the right-left prevalence effect is still unclear.

In the present study, we explored the effect of an internal set on the right-left prevalence effect by using a modification of Meiran's paradigm. We used the difference of compatibility effects along the horizontal and vertical dimensions as an indicator of the right-left prevalence effect. We also analyzed the overall RTs for each dimension (see Proctor et al., 2006) to explore the relationship between the speed of the horizontal and vertical spatial coding and the right-left prevalence (see Hommel, 1996; Rubichi et al., 2004). Faster spatial coding along the structurally salient horizontal dimension should lead to the shorter RTs for a horizontal dimensional task. If this causes the right-left prevalence effect, then the advantage of the horizontal task would covary with the right-left prevalence. However, preparation for each dimension, in addition to the structural salience of the horizontal dimension, may be related to higher horizontal code activation probably accompanying the more efficient suppression of vertical coding (see Meiran, 2000a). If this is essential for the right-left prevalence effect to emerge, then the rightleft prevalence would appear only when participants could prepare for each dimension before the target stimulus presentation, and may not covary with the RT advantage for the horizontal task. In Experiment 1, participants prepared for each dimension in a trial-by-trial manner, and the preparation time was manipulated. In addition to the partial preparation and full preparation conditions, which were included in previous studies (e.g., Meiran, 2005; Proctor et al., 2006), we included a no-preparation condition (i.e., simultaneous presentation of the task cue and target). Experiments 2 and 3 focused on the situation in which the task cue and the target were presented simultaneously because the right-left prevalence effect was absent when there was no preparation time in Experiment 1 (i.e., 0 -msec SOA). In Experiment 2, we replicated Experiment 1 using the simultaneous presentation of the task cue and the target. In Experiment 3, we manipulated participants' set between blocks by changing the relative probability for each task with a 0-msec SOA, in order to dissociate the effects of preparation and the simultaneous presentation of the task cue and the target itself.

\section{EXPERIMENT 1}

We investigated whether the right-left prevalence effect was affected by the preparation for each dimension. For this purpose, vertical and horizontal judgments were intermixed in an unpredictable order. Thus, the task set varied within blocks, and participants responded based on the instructed (i.e., cued) dimension (see Meiran, 1996). We manipulated the preparation time for the upcoming task to test whether the preparation for each dimension differed. We used a sufficient response-cue interval $(1,600 \mathrm{msec}$; see Meiran et al., 2000) to rule out a simple carryover effect because our interest was in the effect of preparation for the task. We used three SOAs (cue-target onset asynchrony): $0 \mathrm{msec}$, during which no preparation could be done; $170 \mathrm{msec}$, during which some but not sufficient preparation could be done; and 1,620 msec, during which sufficient preparation could be done. We chose a 0 -msec SOA in order to include a no-preparation situation. We chose an intermediate SOA of $170 \mathrm{msec}$ and a long SOA of $1,620 \mathrm{msec}$ for two reasons. First, because Meiran (2005) reported no modulation of the right-left prevalence with 200- and 1,200-msec SOAs, we chose an intermediate SOA less than $200 \mathrm{msec}$ but clearly distinct from $0 \mathrm{msec}$, and a long SOA more than 1,200 msec. Second, according to previous studies (e.g., Meiran et al., 2000) and a pilot experiment, it seemed that the preparation for the task proceeds rapidly after the onset of the task cue and then more gradually in this paradigm, so the 170-msec SOA seems to be just under the rapid-preparation phase. 
On the other hand, 1,620 msec would allow enough time to achieve full preparation.

We changed the cue property that might have affected the preparation for each dimension and the resulting right-left prevalence in Meiran's paradigm. Meiran used a pair of arrowheads as the cue. For example, with the horizontal task, the right-pointing arrowhead appeared at the right of the grid, and the left-pointing arrowhead at the left of the grid. It is known that an arrow automatically activates the response corresponding to the pointing direction (Eimer, 1995). Thus the arrowheads might prime the spatial code corresponding to the pointing direction. Similarly, the pair of vertical or horizontal arrowheads might automatically activate the corresponding dimensional task set. These automatic activations of corresponding responses and/or of task-relevant dimensions might affect the right-left prevalence in Meiran's paradigm. To exclude any external spatial information and the spatial asymmetry between the horizontal and the vertical dimensions by task cue, instead of arrowheads we used grid colors as task cues in our experiment. Thus in our experiment, internal preparation for the task, which varies in a trial-by-trial manner, should emerge.

To confirm that participants prepared for the horizontal or vertical dimension in each trial, we included a task switch factor in the analyses. If participants do prepare for each dimension on the basis of the task cue, we would find a cost to switch the task-relevant dimension. Moreover, this switch cost and RT would decrease as the SOA increases. Although these results were obtained in previous task switching studies (e.g., Meiran, 1996), it is necessary to test whether these are replicated in our study despite the methodological differences.

In the $\mathrm{S}-\mathrm{R}$ arrangement used in this study, bimanual responses make the horizontal dimension salient. If the task structure determines the prevalence effect and preparation does not alter the effect (see Proctor et al., 2006; Vu \& Proctor, 2001, 2002), and if the earlier formation of a right-left code elicits the right-left prevalence (see Hommel, 1996; Rubichi et al., 2004), then the right-left prevalence would emerge irrespective of the SOA. On the other hand, if the relative salience of one dimension makes the preparation along that dimension stronger, then the rightleft prevalence would be absent for the simultaneous onset of the cue and target. But the right-left prevalence would increase as the SOA increases.

\section{Method}

Participants. Sixteen students ( 8 female; mean age 22.4 years, range from 19 to 24 years) took part in this experiment. All had normal or corrected-to-normal vision. They were naive as to the purpose of the experiment.

Apparatus and Stimuli. The stimulus presentation and data collection were controlled by an AV-tachistoscope system (Iwatsu ISEL IS-703). Stimuli were presented on a 22 -in. color monitor. A viewing distance of approximately $80 \mathrm{~cm}$ was maintained by a head-andchinrest. The response apparatus consisted of two single key boxes aligned diagonally on a table so that one key was above and to the right, or above and to the left, of the other key, like the pair of keys " 1 " and " 9 " or the pair of keys " 3 " and " 7 on the numeric pad of a keyboard. The two response keys were separated approximately $5 \mathrm{~cm}$ from each other in both the horizontal and vertical dimensions. The right and left keypresses were made with the corresponding index fingers. The vertical dimension of the response apparatus was not aligned parallel to the display but instead projected onto the depth dimension (i.e., the above-below axis corresponded to the far-near axis). However, neither the compatibility effect in the vertical dimension nor the right-left prevalence effect should be influenced by this projection (see Vu et al., 2000). The distance between the center of the response apparatus and the display was approximately $65 \mathrm{~cm}$.

The stimuli were a $2 \times 2$ grid (approximately $3.4^{\circ}$ in visual angle on a side) presented at the center of the display, and a solid circle (approximately $0.4^{\circ}$ in diameter) presented at the center of one of the quadrants of the grid.

Tasks and Procedure. The participants switched between a vertical task and a horizontal task according to the color of the grid. Half of the participants engaged in the vertical task with a green grid and the horizontal task with a red grid, and vice versa for the remaining half. On the vertical task, participants had to press the above or below key as quickly and accurately as possible according to the vertical location of the stimulus regardless of its horizontal location. For example, with the up-left and down-right key configuration, participants had to press the up-left key when the stimulus was presented at the up-right or up-left location, and to press the down-right key when the stimulus was presented at the down-right or down-left location. On the horizontal task, participants had to make a fast and accurate press of the right or left key according to the horizontal location of the stimulus regardless of its vertical location (see Figure 1). Note that the S-R mapping in the task-relevant dimension was always compatible, and the compatibility varied within the task-irrelevant dimension. The horizontal compatibility effect was determined by the difference between the compatible and incompatible conditions of the vertical task (see above-left and belowleft panels of Figure 1, respectively), and the vertical compatibility effect was determined by the difference between the compatible and incompatible conditions of the horizontal task (see above-right and below-right panels of Figure 1, respectively).

The experiment was conducted in a darkened room. Each trial began with the display of the $2 \times 2$ grid in white on a black background for $1,000 \mathrm{msec}$. Then the grid color changed to red or green. With an SOA of 0,170 , or $1,620 \mathrm{msec}$, the target stimulus, a white circle, appeared in the center of one of the four quadrants of the grid until a response was made. As soon as a response was made, all the visual stimuli disappeared. The intertrial interval (ITI) lasted for $600 \mathrm{msec}$. A $500-\mathrm{Hz}$ feedback tone was given during the first $100 \mathrm{msec}$ of the ITI when the wrong key was pressed. Each experimental session consisted of three blocks, and each block consisted of 288 trials of 12 repetitions of the factorial combination of grid color (green or red), SOA $(0,170$, or 1,620 msec), and stimulus location (above-right, above-left, below-right, or below-left) in pseudorandom order. Before the test block, participants completed a practice block consisting of 48 trials. Participants could take short rests between the test blocks.

\section{Results}

The significance criterion was set at $p<.05$ for all analyses. The first trial in each block, trials in which RTs were less than $100 \mathrm{msec}$ or more than $3,000 \mathrm{msec}$ (outliers; $0.05 \%)$, and trials preceded by errors or outliers $(3.0 \%)$ were excluded from all the analyses. After these exclusions, the mean RTs for correct responses and the error rates were submitted to separate ANOVAs with SOA ( 0 , 170 , or $1,620 \mathrm{msec}$ ), task (horizontal or vertical), switch (repeat or switch), and compatibility (compatible or incompatible) as within-participants factors. The mean RTs and error rates for each condition are shown in Table 1.

The ANOVA of the RT data revealed that all four main effects were significant [SOA, $F(2,30)=72.66, p<.001$; 
Table 1

Mean Reaction Times (RTs, in Milliseconds) and Error Rates (\%) for Experiment 1 As a Function of SOA, Task, Switch, and Compatibility

\begin{tabular}{|c|c|c|c|c|}
\hline & \multicolumn{2}{|c|}{ Compatible } & \multicolumn{2}{|c|}{ Incompatible } \\
\hline & RT & Error Rate & RT & Error Rate \\
\hline \multicolumn{5}{|c|}{$\mathrm{SOA}=0 \mathrm{msec}$} \\
\hline \multicolumn{5}{|c|}{ Horizontal Task } \\
\hline Repeat & 543 & 0.0 & 691 & 4.5 \\
\hline Switch & 617 & 0.4 & 792 & 6.0 \\
\hline \multicolumn{5}{|c|}{ Vertical Task } \\
\hline Repeat & 566 & 0.2 & 720 & 4.7 \\
\hline Switch & 672 & 0.9 & 863 & 11.4 \\
\hline \multicolumn{5}{|c|}{$\mathrm{SOA}=170 \mathrm{msec}$} \\
\hline \multicolumn{5}{|c|}{ Horizontal Task } \\
\hline Repeat & 509 & 0.0 & 627 & 2.4 \\
\hline Switch & 545 & 0.2 & 699 & 6.0 \\
\hline \multicolumn{5}{|c|}{ Vertical Task } \\
\hline Repeat & 521 & 0.0 & 682 & 6.0 \\
\hline Switch & 580 & 0.0 & 804 & 9.4 \\
\hline \multicolumn{5}{|c|}{$\mathrm{SOA}=1,620 \mathrm{msec}$} \\
\hline \multicolumn{5}{|c|}{ Horizontal Task } \\
\hline Repeat & 452 & 0.4 & 536 & 1.9 \\
\hline Switch & 452 & 0.4 & 562 & 4.3 \\
\hline \multicolumn{5}{|c|}{ Vertical Task } \\
\hline Repeat & 480 & 0.2 & 629 & 4.7 \\
\hline Switch & 475 & 0.0 & 645 & 8.6 \\
\hline
\end{tabular}

task, $F(1,15)=9.28, p<.01$; switch, $F(1,15)=115.44$, $p<.001$; compatibility, $F(1,15)=31.10, p<.001]$. Response latency decreased as SOA increased $(683,621$, and $589 \mathrm{msec}$ for SOAs of 0,170 , and 1,620 msec, respectively). Participants responded faster on the horizontal task (585 $\mathrm{msec})$ than on the vertical task (636 msec). RTs were shorter when participants repeated tasks $(580 \mathrm{msec})$ than when they shifted tasks (642 msec). Responses were faster with compatible trials (534 msec) than with incompatible trials (687 $\mathrm{msec})$.

The two-way interaction between SOA and switch was significant $[F(2,30)=35.04, p<.001]$. The 106-msec switch cost with a simultaneous onset of task cue and a

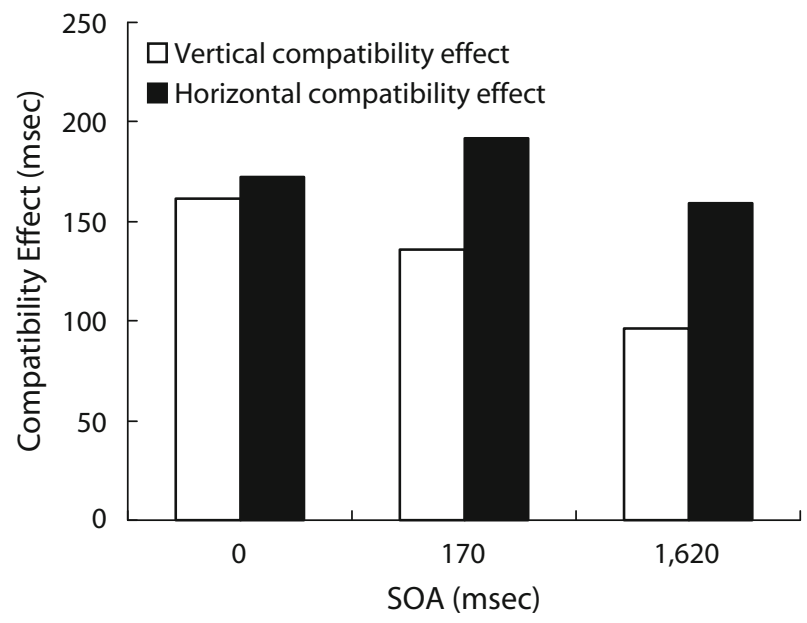

Figure 2. Compatibility effects for Experiment 1 as a function of dimension and SOA. target decreased to $72 \mathrm{msec}$ with the intermediate SOA, and finally almost disappeared $(9 \mathrm{msec})$ with the longest SOA. The three-way interaction between SOA, task, and switch was significant $[F(2,30)=3.48, p<.05]$. Switch costs were larger with the vertical task than with the horizontal task only when the SOA was $0 \mathrm{msec}$ (125 vs. $87 \mathrm{msec}$ ) or $170 \mathrm{msec}$ (90 vs. $54 \mathrm{msec}$ ). The switch cost almost disappeared when the SOA was $1,620 \mathrm{msec}$ for both tasks ( 5 vs. $12 \mathrm{msec}$ ). The significant two-way interaction between switch and compatibility $[F(1,15)=16.33$, $p<.005$ ] revealed a larger switch cost in the incompatible condition $(80 \mathrm{msec})$ as compared to the compatible condition (45 msec).

The significant two-way interaction between SOA and compatibility $[F(2,30)=4.22, p<.05]$ indicated a smaller compatibility effect with the $1,620-\mathrm{msec}$ SOA (128 msec) than with $0-\mathrm{msec}$ and $170-\mathrm{msec}$ SOAs (167 and $164 \mathrm{msec}$, respectively). Most importantly, the threeway interaction between SOA, task, and compatibility was significant $[F(2,30)=4.45, p<.05]$. As shown in Figure 2, the right-left prevalence increased as the SOA increased. The magnitudes of the right-left prevalence effect were $11 \mathrm{msec}$ (n.s.), $56 \mathrm{msec}(p=.062)$, and $63 \mathrm{msec}$ $(p<.05)$ for each SOA. The right-left prevalence effects did not statistically differ in size for the latter two SOAs. No other interactions were significant.

The ANOVA of error rates revealed a main effect of SOA $[F(2,30)=3.69, p<.05]$. Error rates decreased as SOA increased $(3.5 \%, 3.0 \%$, and $2.6 \%$ for SOAs of 0,170 , and 1,620 $\mathrm{msec}$, respectively). The main effect of task was significant $[F(1,15)=9.03, p<.01]$. Participants responded more accurately to the horizontal task $(2.2 \%)$ than to the vertical task $(3.8 \%)$. The main effect of switch $[F(1,15)=17.94, p<.001]$ revealed more errors on switch trials $(4.0 \%)$ than on repeat trials $(2.1 \%)$. The main effect of compatibility was significant $[F(1,15)=$ $30.36, p<.001]$. Participants made few errors on compatible trials $(0.2 \%)$, and made most of the errors on incompatible trials $(5.8 \%)$.

All the two-way interactions involving compatibility were significant: with $\mathrm{SOA}, F(2,30)=4.06, p<.05$; with task, $F(1,15)=8.57, p<.05$; and with switch, $F(1,15)=$ $15.85, p<.005$. These reflected differences in accuracy as shown by main effect of each factor, which appeared only in the incompatible condition because of a floor effect in the compatible condition (see Table 1). The other interactions were not significant.

\section{Discussion}

With regard to the task switching, our results generally replicated the findings in previous studies (see Meiran, 2000a). With increasing preparation time, tasks were performed more efficiently. We obtained switch costs that decreased as the preparation time for the upcoming task increased. Although the "residual" switch cost at the 1,620-msec SOA was not significant in this experiment, a residual cost is not always present in task switching studies and is affected by some factors (see, e.g., De Jong, 2000; González, Milán, Pereda, \& Hochel, 2005; Meiran, 2000b; Meiran \& Chorev, 2005). In contrast to the absence 
of a residual cost in our study, Proctor et al. (2006, Experiment 3) found a constant switching cost across SOAs using a similar switching procedure. Thus, the effect of preparation on switching cost in Meiran's task switching paradigm seems to be unstable. Some methodological factor(s) might affect the residual cost in our experiment, but this issue is beyond the scope of our study. The compatibility effect was present, and the switch cost was larger in the incompatible condition than in the compatible condition. The three-way interaction of switch, SOA, and compatibility was not significant. Unlike Meiran's (1996) previous study, the horizontal task was easier than the vertical task (but see Meiran et al., 2000).

The right-left prevalence effect was absent when there was no preparation time for the upcoming task. However, the prevalence effect increased as the SOA increased, although the compatibility effect itself decreased as the SOA increased. These results contradict the faster horizontal code formation hypothesis (Hommel, 1996; Rubichi et al., 2004) that would predict an unchanged rightleft prevalence effect across SOAs because the horizontal code should have been formed faster in the task structure of this experiment where participants used right and left hands as response effectors. Rather, the results suggest that preparation for the horizontal dimension would be stronger than for the vertical dimension when participants preliminarily prepared for each dimension.

Our results show the effect of SOA on the right-left prevalence effect, which Meiran (2005) did not find. Although this would appear to be a discrepancy between these two studies, the results are consistent when the SOAs used in both studies are considered. We obtained no prevalence with a 0 -msec SOA, a 56-msec nonsignificant tendency of the prevalence with a $170-\mathrm{msec}$ SOA, and a $63-\mathrm{msec}$ significant right-left prevalence with a 1,620 -msec SOA. The SOAs used in Meiran (2005) were $200 \mathrm{msec}$ and $1,200 \mathrm{msec}$, both of which were between our middle SOA and long SOA. The right-left prevalence effect of $58 \mathrm{msec}$ obtained by Meiran (2005) was numerically between the prevalence effect with the $170-\mathrm{msec}$ SOA and that with the 1,620 -msec SOA in our experiment. Thus, the time course of the right-left prevalence effect found in Meiran (2005) is consistent with our experimental findings.

Proctor et al. (2006) reported that preparation for the upcoming task did not affect the right-left prevalence. This is inconsistent with the results obtained in our experiment. However, the results of both experiments show some consistency. Unlike the usual right-left prevalence studies, Proctor et al. (2006) used the RTs for each task as indicators of the prevalence effect, and found that RTs for the horizontal task were faster than for the vertical task. However, the SOA had little effect on this horizontal task superiority, which was found in our experiment as well. On the other hand, the right-left prevalence effect (superiority of the horizontal compatibility effect to the vertical compatibility effect) in our experiment increased as the preparation time increased. In Proctor et al. (2006, Experiment 3), no significant effect of SOA on this rightleft prevalence was obtained although the right-left prevalence effect was numerically smaller for a short SOA of
$100 \mathrm{msec}$ than a long SOA of $900 \mathrm{msec}$. This is probably because of the differences of the shortest SOA adopted in each experiment. We used $0 \mathrm{msec}$, whereas they used $100 \mathrm{msec}$. Overall, an increasing right-left prevalence as a function of SOA would be the pattern. The nonsignificant effect in Proctor et al. (2006) indicates that the preparation for a task that elicits the prevalence effect proceeds rapidly. In fact, the present study showed little, if any, increase in the right-left prevalence effect from the 170- to the 1,620-msec SOA. The difference of RTs and the compatibility effect for horizontal and vertical dimensions as a function of preparation time provide some indication about the effect of the relative salience on dimensional processing and right-left prevalence. We discuss this issue more in the General Discussion.

\section{EXPERIMENT 2}

The failure to find a right-left prevalence effect for the 0 -msec SOA in Experiment 1 seems to be unique and theoretically important. To test the generality of the absence of the right-left prevalence effect when there is no preparation time for each dimension, in Experiment 2 we replicated Experiment 1 using only the simultaneous presentation of the task cue and target. We changed the task cue so that the task cue and the relevant target information were conveyed by the same stimulus. Although the vertical and the horizontal tasks were the same as in Experiment 1, the target shape signaled the to-be-conducted task. Because all of the trials involved 0-msec SOA, participants were aware that they would have no time to prepare after cue onset.

\section{Method}

Participants. Twelve students ( 6 female; mean age 22.0 years, range from 21 to 26 years) participated. All had normal or correctedto-normal vision. They were naive to the purpose of the experiment. None of them had participated in Experiment 1.

Apparatus, Stimuli, Tasks, and Procedure. These were the same as in Experiment 1, except for the differences noted below. The SOA was fixed at $0 \mathrm{msec}$. The target stimulus was a solid circle (approximately $0.4^{\circ}$ in diameter) or a solid square (approximately $0.4^{\circ}$ on a side) at the center of one of the quadrants of the grid. All of the stimuli were presented in white on a black background. The participants switched between a vertical task and a horizontal task according to the stimulus shape. Half of the participants conducted the vertical task with a circle stimulus and the horizontal task with a square stimulus, and vice versa for the remaining half. Each experimental session consisted of four blocks, and each block consisted of 128 trials of 16 repetitions of the factorial combination of stimulus shape (square or circle) and stimulus location (above-right, aboveleft, below-right, or below-left) in pseudorandom order. Before the test block, participants engaged in a practice block consisting of 64 trials.

\section{Results}

The first trial in each block, trials in which the RTs were less than $100 \mathrm{msec}$ or more than 3,000 msec (outliers; $0.03 \%)$, and trials preceded by errors or outliers $(4.1 \%)$ were excluded from all the analyses. After this exclusion, the mean RTs for correct responses and the error rates were submitted to separate ANOVAs with task (horizontal or vertical), switch (repeat or switch), and compatibility 
Table 2

Mean Reaction Times (RTs, in Milliseconds) and Error Rates (\%) for Experiment 2 As a Function of Task, Switch, and Compatibility

\begin{tabular}{lccccc}
\hline & \multicolumn{2}{c}{ Compatible } & & \multicolumn{2}{c}{ Incompatible } \\
\cline { 2 - 3 } \cline { 6 - 6 } & RT & Error Rate & & RT & Error Rate \\
\hline Horizontal Task & & & & & \\
$\quad$ Repeat & 476 & 0.0 & & 750 & 3.9 \\
$\quad$ Switch & 508 & 0.3 & & 813 & 7.9 \\
Vertical Task & & & & \\
$\quad$ Repeat & 529 & 0.8 & & 851 & 11.2 \\
Switch & 568 & 0.8 & & 870 & 10.6 \\
\hline
\end{tabular}

(compatible or incompatible) as within-participants factors. The mean RTs and error rates for each condition are shown in Table 2.

An ANOVA of the RT data revealed main effects of task $[F(1,11)=6.30, p<.05]$, switch $[F(1,11)=6.34$, $p<.05]$, and compatibility $[F(1,11)=34.70, p<.01]$. Responses were faster for the horizontal task $(637 \mathrm{msec})$ than for the vertical task $(705 \mathrm{msec})$, for the repeat trials $(652 \mathrm{msec})$ than for the switch trials $(690 \mathrm{msec})$, and for the compatible condition $(520 \mathrm{msec})$ than for the incompatible condition $(821 \mathrm{msec})$. No interactions were significant, including the two-way interaction between task and compatibility $[F(1,11)<1, p=.37]$. The vertical compatibility effect $(290 \mathrm{msec})$ was comparable to the horizontal compatibility effect $(312 \mathrm{msec})$.

An ANOVA of the error rate data revealed a main effect of task $[F(1,11)=5.69, p<.05]$. Participants responded more accurately to the horizontal task (3.0\%) than to the vertical task $(5.8 \%)$. The main effect of compatibility $[F(1,11)=15.40, p<.01]$ revealed more errors on incompatible trials $(8.4 \%)$ than on compatible trials $(0.4 \%)$. No other main effect or interactions were significant.

\section{Discussion}

Although RTs for the horizontal task were faster than for the vertical task (see also Proctor et al., 2006), the compatibility effects along the horizontal and vertical dimensions did not differ significantly. Despite the methodological differences between Experiments 1 and 2, we successfully replicated the absence of the right-left prevalence effect for a 0 -msec cue-target SOA. This indicates that the disappearance of the right-left prevalence with simultaneous presentation of task cue and target is a replicable general phenomenon and not due to the mixing of longer SOAs in Experiment 1. We conclude that the right-left prevalence effect does not occur without preparation for the upcoming task dimension.

\section{EXPERIMENT 3}

Experiment 1 revealed a right-left prevalence for task set preparation preceding the target onset within blocks. However, the right-left prevalence effect was absent when there was no time for preparation for the task in Experiments 1 and 2. The absence of the right-left prevalence effect with a 0 -msec SOA might be due not to the absence of preparation for the task dimension, but to the simulta- neous presentation of the task cue and target. To exclude this possibility, we conducted an experiment with simultaneous presentation of the task cue and target, but with overall preparation for each dimension. We used a 0 -msec SOA only, and manipulated the relative frequency of each task in a block.

Manipulation of task frequency is similar to the manipulation of instructions in the right-left prevalence research literature (e.g., Hommel, 1996; Vu et al., 2000) in that it affects the internal set of the participants beyond each trial. If participants prepare for the frequent task, then the RTs should be faster for the frequent task than for the infrequent task. In such a case, if the right-left prevalence comes from the difference of the preparation for each dimension, then the compatibility effect advantages along the frequent task dimension and the right-left prevalence effect when collapsed across the horizontal frequent and vertical frequent conditions should appear analogous to the effect of instruction (see, e.g., Hommel, 1996; Vu et al., 2000). On the other hand, if the absence of the right-left prevalence in Experiments 1 and 2 were due to the simultaneous presentation of task cue and target, then no right-left prevalence should be obtained in this experiment either.

\section{Method}

Participants. Sixteen students ( 8 female; mean age 21.8 years, range from 19 to 24 years) took part in this experiment. All had normal or corrected-to-normal vision. They were naive as to the purpose of the experiment. None of them had participated in previous experiments of this study.

Apparatus, Stimuli, Tasks, and Procedure. These were the same as in Experiment 1, except for the differences noted below. The SOA was $0 \mathrm{msec}$ in all the trials. We manipulated the probability of each task between blocks. Half of the participants performed one practice and two experimental blocks with $80 \%$ green grids and $20 \%$ red grids, and then performed one practice and two experimental blocks with $80 \%$ red grids and $20 \%$ green grids. The other half performed the reversed order of task frequency. Each practice block consisted of 80 trials and each test block consisted of 180 trials. Participants were explicitly informed which task was frequent in the subsequent blocks before each task frequency condition started.

\section{Results}

The first trial in each block, trials in which RTs were less than $100 \mathrm{msec}$ or more than 3,000 msec (outliers; $0.03 \%)$, and trials preceded by errors or outliers $(3.2 \%)$ were excluded from all the analyses. After this exclusion, the mean RTs for correct responses and the error rates were submitted to separate ANOVAs with frequent task (horizontal or vertical), task (horizontal or vertical), and compatibility (compatible or incompatible) as withinparticipants factors. Because the number of trials with the repeat condition of the infrequent task was small, and because task switch did not modulate the right-left prevalence in Experiments 1 and 2, we did not use switch as a factor in Experiment 3. The mean RTs and error rates for each condition are shown in Table 3.

The main effect of frequent task $[F(1,15)=4.66, p<$ $.05]$ indicated that RTs were faster when the frequent task was the horizontal task $(523 \mathrm{msec})$ rather than the vertical task $(547 \mathrm{msec})$. The two-way interaction between 
Table 3

Mean Reaction Times (RTs, in Milliseconds) and Error Rates (\%) for Experiment 3 As a Function of Frequent Task, Task, and Compatibility

\begin{tabular}{lccccc}
\hline & \multicolumn{2}{c}{ Compatible } & & \multicolumn{2}{c}{ Incompatible } \\
\cline { 2 - 3 } \cline { 6 - 7 } & RT & Error Rate & & RT & Error Rate \\
\hline Horizontal Frequent* & & & & & \\
$\quad$ Horizontal task & 387 & 0.4 & & 461 & 4.4 \\
$\quad$ Vertical task & 546 & 0.2 & & 701 & 12.8 \\
Vertical Frequent* & & & & \\
$\quad$ Horizontal task & 552 & 0.3 & & 701 & 11.1 \\
$\quad$ Vertical task & 407 & 0.4 & & 526 & 6.3 \\
\hline
\end{tabular}

${ }^{*}$ Frequent means $80 \%$ of the trials.

frequent task and task $[F(1,15)=138.56, p<.001]$ showed faster responses for the frequent task. The horizontal task was responded to faster than the vertical task when the horizontal task was frequent (424 vs. $623 \mathrm{msec}$ ), whereas the opposite was true when the vertical task was frequent (627 vs. $467 \mathrm{msec}$ ). The main effect of compatibility $[F(1,15)=79.04, p<.001]$ revealed a $124-\mathrm{msec}$ compatibility effect. The three-way interaction of frequent task, task, and compatibility $[F(1,15)=8.87, p<.01]$ revealed an advantage of compatibility effect along dimension for the frequent task (see Figure 3). We obtained an 81-msec right-left advantage when the horizontal task was frequent, and a 29-msec top-bottom advantage when the vertical task was frequent. The significant interaction of task and compatibility $[F(1,15)=8.87, p<.01]$ indicated an overall right-left prevalence. This reflects that the right-left advantage for the horizontal task frequent situation was larger than the top-bottom advantage for the vertical task frequent situation. No other main effects or interactions were significant.

An ANOVA of error rates revealed a main effect of compatibility $[F(1,15)=50.56, p<.001]$. Participants made few errors on compatible trials $(0.3 \%)$, and made almost all of the errors on incompatible trials (8.6\%). The two-way interaction between frequent task and task $[F(1,15)=11.84, p<.005]$ indicated fewer errors on the

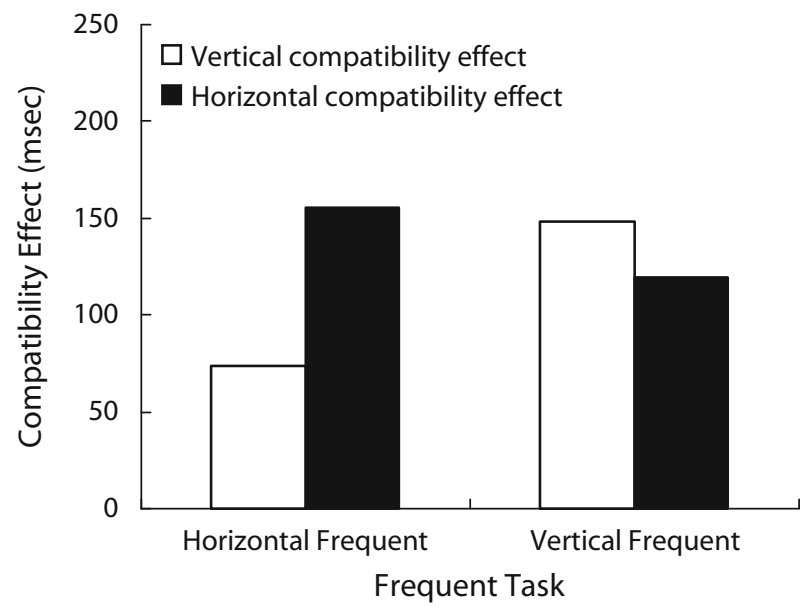

Figure 3. Compatibility effects for Experiment 3 as a function of dimension and frequent task. frequent task (2.9\%) than on the infrequent task (6.1\%). The horizontal task was less error prone than the vertical task when the horizontal task was frequent $(2.4 \% \mathrm{vs}$. $6.5 \%$ ), whereas the opposite was true when the vertical task was frequent (5.7\% vs. $3.4 \%)$. The three-way interaction between frequent task, task, and compatibility was significant $[F(1,15)=20.44, p<.001]$, indicating the advantage of the compatibility effect along the dimension for the frequent task. We obtained an $8.5 \%$ right-left advantage when the horizontal task was frequent, and a $4.9 \%$ top-bottom advantage when the vertical task was frequent. Other main effects or interactions were not significant.

\section{Discussion}

Performance was better for the frequent task, confirming that the participants prepared for the frequent task. The compatibility effect for the dimension of the frequent task was larger than for the dimension of the infrequent task. The difference of participants' mental sets between blocks led to an advantage for the prepared dimension. Most importantly, we obtained the right-left prevalence effect. The right-left advantage in the horizontal frequent condition was larger than the top-bottom advantage in the vertical frequent condition. Right-left prevalence did not emerge when the SOA was 0 msec in Experiments 1 and 2. In those experiments, the probability for the tasks was equal, which should have eliminated participants' preparation at the $0-\mathrm{msec}$ SOA. In contrast, in Experiment 3 , the probability of each task was not equal and participants seemed to prepare for the frequent task, thus already activating a set for the dimension of the frequent task before the task was specified. Thus, the right-left prevalence effect emerged when participants prepared for each dimension even with a 0 -msec SOA. This indicates that the absence of the prevalence effect with a 0 -msec SOA in Experiments 1 and 2 was due to the absence of preparation for each dimension, not to the simultaneous presentation of the task cue and target.

The findings in this experiment were very similar to the findings in research studies using instruction manipulation. The right-left advantage with horizontal instruction was larger than the top-bottom advantage with vertical instruction in many of the right-left prevalence studies in which participants prepared for the instructed dimension prior to the target presentation (e.g., Hommel, 1996; Vu et al., 2000). Considering the instruction and frequency effects, it seems that people can prepare for either the horizontal or the vertical dimension, but that preparation for the horizontal dimension can be more powerful, leading to the right-left prevalence.

\section{GENERAL DISCUSSION}

In this study, we investigated the effect of participants' preparation for vertical and horizontal dimensions on right-left prevalence. In Experiment 1, we tested the effect of preparation for horizontal and vertical coding on right-left prevalence and its time course in a trial-by-trial manner. The vertical and horizontal tasks were mixed within a block, and the upcoming task was specified by 
a task cue simultaneously with or preceding the target. When the participants did not prepare for the task (i.e., the simultaneous presentation of the cue and the target), no prevalence effect was observed. However, the right-left prevalence effect increased as the SOA increased. Experiment 2 replicated the absence of the right-left prevalence effect in the absence of preparation for the upcoming task by using simultaneous presentation of the task cue and the target. In Experiment 3, we dissociated the preparation for each dimension and the simultaneous presentation of the task cue and the target by manipulating the frequency of each task between blocks while keeping the SOA at $0 \mathrm{msec}$. Although we obtained advantages for the dimension of the frequent task, we obtained an overall right-left prevalence effect when the findings were collapsed across the horizontal and vertical frequent conditions. Thus, we showed the importance of preparation for the horizontal and vertical dimensions for the right-left prevalence effect.

The horizontal dimension is more salient than the vertical dimension in the task structure of this study (bimanual responses using two proximally separated diagonal keys on a table and the stimuli equally separated along the horizontal and vertical dimensions), probably due to the use of right and left effectors (see Vu \& Proctor, 2001). Hommel (1996) also indicated the importance of the use of right and left effectors in right-left prevalence. One might argue that the response arrangement would also play a role in determining the relative salience. Responses were not placed one above the other but rather away from or near the body in this study; we regarded this depth dimension as the vertical dimension. This projection might decrease the compatibility effect for the vertical dimension because the vertical responses in fact do not vary along that dimension. However, $\mathrm{Vu}$ et al. (2000) showed that neither the compatibility effect in the vertical dimension nor the right-left prevalence effect was influenced by this projection. Moreover, the right-left prevalence effect was obtained even with responses that varied in height (see, e.g., Hommel, 1996; Nicoletti \& Umiltà, 1984; Vu et al., 2000). Thus, the projection of the vertical dimension on the depth dimension in the arrangement of the response keys seems not to affect the task structure that determines the relative salience.

The relative salience of the horizontal dimension determined by the task structure seems to exert two separate effects on performance. One is an overall superiority for the horizontal task in comparison with the vertical task (see also Proctor et al., 2006), and the other is the rightleft prevalence (larger horizontal compatibility relative to vertical compatibility). Although the horizontal task superiority was present in all of the experiments in this study, the right-left prevalence effect emerged only when participants prepared for each dimension prior to the target presentation. The horizontal task superiority indicates that the formation of right and left codes was always faster than the formation of top and bottom codes. Hommel (1996) and Rubichi et al. (2004) proposed that faster horizontal coding than vertical coding elicits the right-left prevalence. If this hypothesis were accurate, we should have obtained a right-left prevalence regardless of the SOA in Experiments 1 and 2. In fact, the use of right and left effectors seemed to accelerate performance on the horizontal task itself, but the faster horizontal coding was not enough to elicit a right-left prevalence effect. The right-left prevalence emerged only when participants prepared for horizontal or vertical dimensions in advance.

The importance of preparation for right-left prevalence to occur is consistent with recent findings obtained in right-left prevalence studies with Simon effects in which the stimulus position was irrelevant to the task. The rightleft prevalence effect was not observed in Simon tasks with bimanual keypress responses (Proctor et al., 2003; $\mathrm{Vu}$ et al., 2005). The absence of the right-left prevalence effect with Simon effects, in which participants do not intentionally prepare for spatial stimulus properties, also supports our hypothesis that the salience of the horizontal dimension, based on the use of right and left effectors, affects the right-left prevalence only when participants prepare for each dimension. Rubichi, Nicoletti, and Umiltà (2005) obtained the right-left prevalence effect in the Simon task with hand and foot responses. The response arrangement used in Rubichi et al. (2005) might make the horizontal dimension more salient than that used for bimanual keypress responses because of the larger response key distance (Vu et al., 2000). Alternatively, the use of the hand and foot itself may affect the relative salience of each dimension. Thus, as a result, the relative salience of the horizontal dimension may lead to the larger Simon effect for the horizontal dimension (Proctor et al., 2003) in Rubichi et al. (2005).

How does the greater preparation for the salient dimension lead to the right-left prevalence? One possibility is that faster formation of spatial codes along the salient dimension elicits the right-left prevalence (see also Hommel, 1996; Rubichi et al., 2004). Although shorter RTs for horizontal tasks confirmed the faster spatial coding along the salient dimension in this study, this faster coding did not always lead to the right-left prevalence effect. We speculate that the greater preparation for the horizontal dimension yields higher activation of the horizontal codes than the vertical codes, eliciting a larger horizontal compatibility effect (i.e., right-left prevalence effect). Furthermore, greater activation of the stimulus code for one dimension might lead to more efficient suppression of the stimulus code for another dimension (see Meiran, 2000a).

Adam, Hommel, and Umiltà (2003, 2005) have described a right-left advantage for selecting groups of responses. In their study, two of four horizontally aligned responses were cued in advance. The cuing of the two rightmost or leftmost responses was effective relative to the inner-outer or the alternate-two response cuing, and this right-left advantage tended to disappear as the level of preparation increased. Here the right-left advantage/ prevalence did not depend on the level of preparation or increase as a function of the level of preparation. In Adam et al. (2003, 2005), the right-left advantage was related to the preparation for each response group within the horizontal dimension. The right-left prevalence effect 
was related to the advantage of the horizontal dimension relative to the vertical dimension when participants prepared for each dimension. Thus, the right-left advantage in response cuing and the right-left prevalence in twodimensional S-R compatibility seem to reflect different mechanisms. The effect of preparation on the right-left prevalence effect seems to be specific to the relationship between the horizontal and the vertical dimensions.

In our research paradigm, the compatibility effect based on the spatial correspondence between the stimulus location and the response location along the task-irrelevant dimension was confounded with the congruency effect based on the correspondence of the responses between the relevant and the irrelevant tasks, which has usually been observed in task switching studies. However, the congruency effect alone cannot explain the results in our study. In our Experiment 1, the compatibility effect was affected by the SOA, and its time course differed between the horizontal and the vertical tasks, although the congruency effect is generally unaffected by the SOA (Sudevan \& Taylor, 1987). One might argue that the compatibility effect should be absent when the compatible and the incompatible mappings are mixed (e.g., Shaffer, 1965). However, in our experiment, the mapping was always compatible and the compatibility varied only for the irrelevant dimension. The task-relevance of each dimension varied within a block. When spatially irrelevant tasks were mixed with spatially relevant tasks with compatible S-R mapping, the spatial compatibility effect for an irrelevant dimension (i.e., the Simon effect) increased (Marble \& Proctor, 2000). Thus, the probability for compatibility effects to emerge still existed in our experiment.

Meiran (2005) conducted an experiment with the compatible mapping condition and the incompatible mapping condition on a relevant dimension. He predicted that the direction of the compatibility effect (on an irrelevant dimension) and the direction of the congruency effect should be opposite in the incompatible mapping condition. He found that the congruency effects did not differ in magnitude for both mapping conditions, and concluded that the congruency effect was dominant in this paradigm. However, his prediction was problematic. When the task in the relevant dimension requires reverse mapping, the reverse rule is also applied to the irrelevant spatial dimension, leading to a reversed Simon effect (logical recoding; Hedge \& Marsh, 1975). Considering the logical recoding, the irrelevant compatibility effect in the incompatible mapping condition might work in the direction opposite to the direction of the irrelevant compatibility effect in the compatible mapping condition. Thus, the direction of the compatible effect and the congruency effect might be the same even in the incompatible mapping condition. The results of Meiran (2005) could not exclude the existence of compatibility effect in this paradigm. Taken together, the compatibility effect should have emerged in our study.

The right-left prevalence effect emerged only when participants prepared for each dimension in advance of the target presentation. This indicates the essential role of internal mental set in the right-left prevalence effect. In contrast, the horizontal task superiority was observed re- gardless of the preparatory state. These findings indicate that the relative salience of the horizontal dimension determined by the task structure leads to faster horizontal coding and stronger preparation for the horizontal dimension than for the vertical dimension. Although the former does not directly elicit the prevalence effect, the latter would elicit higher activation of the horizontal code, resulting in the right-left prevalence effect. The right-left prevalence cannot be fully explained solely by the relative salience determined by the task structure (see, e.g., Proctor et al., 2006) or by faster horizontal coding (e.g., Hommel, 1996; Rubichi et al., 2004). The internal preparatory state would play a critical role in the right-left prevalence.

\section{AUTHOR NOTE}

This research was supported by a grant from the research fellowships of the Japan Society for the Promotion of Science for Young Scientists to A.N. and by a grant-in-aid for scientific research from the Japan Society for the Promotion of Science awarded to K.Y. We thank Shinya Takahashi for his assistance with data collection, and Dale Dagenbach, Robert W. Proctor, and two anonymous reviewers for their helpful and constructive comments. Correspondence related to this article may be sent to A. Nishimura, Department of Psychology, Graduate School of Humanities and Sociology, University of Tokyo, 7-3-1 Hongo, Bunkyo-ku, Tokyo 113-0033, Japan (e-mail: akio@L.u-tokyo.ac.jp).

\section{REFERENCES}

Adam, J. J., Hommel, B., \& Umiltà, C. (2003). Preparing for perception and action (I): The role of grouping in the response-cuing paradigm. Cognitive Psychology, 46, 302-358.

Adam, J. J., Hommel, B., \& Umiltà, C. (2005). Preparing for perception and action (II): Automatic and effortful processes in response cueing. Visual Cognition, 12, 1444-1473.

BREBNER, J. (1973). S-R compatibility and changes in RT with practice. Acta Psychologica, 37, 93-106.

DE Jong, R. (2000). An intention-activation account of residual switch costs. In S. Monsell \& J. Driver (Eds.), Control of cognitive processes: Attention and performance XVIII (pp. 357-376). Cambridge, MA: MIT Press.

EIMER, M. (1995). Stimulus-response compatibility and automatic response activation: Evidence from psychophysiological studies. Journal of Experimental Psychology: Human Perception \& Performance, 21, 837-854.

González, A., Milán, E. G., Pereda, A., \& Hochel, M. (2005). The response-cued completion hypothesis and the nature of residual cost in regular switch. Acta Psychologica, 120, 327-341.

Hedge, A., \& Marsh, N. W. A. (1975). The effect of irrelevant spatial correspondences on two-choice reaction time task. Acta Psychologica, 39, 427-439.

Hommel, B. (1996). No prevalence of right-left over top-bottom spatial codes. Perception \& Psychophysics, 58, 102-110.

Kornblum, S., HasbroucQ, T., \& Osman, A. (1990). Dimensional overlap: Cognitive basis for stimulus-response compatibility-A model and taxonomy. Psychological Review, 97, 253-270.

Lu, C.-H., \& Proctor, R. W. (1995). The influence of irrelevant location information on performance: A review of the Simon and spatial Stroop effects. Psychonomic Bulletin \& Review, 2, 174-207.

Marble, J. G., \& Proctor, R. W. (2000). Mixing location-relevant and location-irrelevant choice-reaction tasks: Influences of location mapping on the Simon effect. Journal of Experimental Psychology: Human Perception \& Performance, 26, 1515-1533.

Meiran, N. (1996). Reconfiguration of processing mode prior to task performance. Journal of Experimental Psychology: Learning, Memory, \& Cognition, 22, 1423-1442.

Meiran, N. (2000a). Modeling cognitive control in task-switching. Psychological Research, 63, 234-249.

MeIran, N. (2000b). Reconfiguration of stimulus task sets and response task sets during task switching. In S. Monsell \& J. Driver (Eds.), Con- 
trol of cognitive processes: Attention and performance XVIII (pp. 377399). Cambridge, MA: MIT Press.

Meiran, N. (2005). Task rule-congruency and Simon-like effects in switching between spatial tasks. Quarterly Journal of Experimental Psychology, 58A, 1023-1041.

Meiran, N., \& Chorev, Z. (2005). Phasic alertness and the residual task-switching cost. Experimental Psychology, 52, 109-124.

Meiran, N., Chorev, Z., \& SAPIR, A. (2000). Component processes in task switching. Cognitive Psychology, 41, 211-253.

Nicoletti, R., \& Umiltà, C. (1984). Right-left prevalence in spatial compatibility. Perception \& Psychophysics, 35, 333-343.

Nicoletti, R., \& Umiltì, C. (1985). Responding with hand and foot: The right/left prevalence in spatial compatibility is still present. Perception \& Psychophysics, 38, 211-216.

Nicoletti, R., Umiltà, C., Tressoldi, E. P., \& Marzi, C. A. (1988). Why are left-right spatial codes easier to form than above-below ones? Perception \& Psychophysics, 43, 287-292.

Proctor, R. W., Косн, I., \& VU, K.-P. L. (2006). Effects of precuing horizontal and vertical dimensions on right-left prevalence. Memory \& Cognition, 34, 949-958.

Proctor, R. W., \& Reeve, T. G. (Eds.) (1990). Stimulus-response compatibility: An integrated perspective. Amsterdam: North-Holland.

Proctor, R. W., Vu, K.-P. L., \& Nicoletti, R. (2003). Does right-left prevalence occur for the Simon effect? Perception \& Psychophysics, 65, 1318-1329.

Rubichi, S., Nicoletti, R., Pelosi, A., \& Umiltà, C. (2004). Rightleft prevalence effect with horizontal and vertical effectors. Perception \& Psychophysics, 66, 255-263.

Rubichi, S., Nicoletti, R., \& Umiltà, C. (2005). Right-left prevalence with task-irrelevant spatial codes. Psychological Research, 69 , 167-178.

Rubichi, S., Vu, K.-P. L., Nicoletti, R., \& Proctor, R. W. (2006). Spatial coding in two dimensions. Psychonomic Bulletin \& Review, 13, 201-216.

ShafFer, L. H. (1965). Choice reaction with variable S-R mapping. Journal of Experimental Psychology, 70, 284-288.

Simon, J. R. (1990). The effects of an irrelevant directional cue on human information processing. In R. W. Proctor \& T. G. Reeve (Eds.), Stimulus-response compatibility: An integrated perspective (pp. 31-86). Amsterdam: North-Holland.

Simon, J. R., \& Craft, J. L. (1972). Reaction time in an oddity task:
Responding to the "different" element of a three-light display. Journal of Experimental Psychology, 92, 405-411.

SudEVAN, P., \& TAYLOR, D. A. (1987). The cuing and priming of cognitive operations. Journal of Experimental Psychology: Human Perception \& Performance, 13, 89-103.

Umiltà, C., \& Nicoletti, R. (1990). Spatial stimulus-response compatibility. In R. W. Proctor \& T. G. Reeve (Eds.), Stimulus-response compatibility: An integrated perspective (pp. 89-116). Amsterdam: North-Holland.

Vu, K.-P. L., Pellicano, A., \& Proctor, R. W. (2005). No overall right-left prevalence for horizontal and vertical Simon effects. Perception \& Psychophysics, 67, 929-938.

Vu, K.-P. L., \& Proctor, R. W. (2001). Determinants of right-left and top-bottom prevalence for two-dimensional spatial compatibility. Journal of Experimental Psychology: Human Perception \& Performance, 27, 813-828.

Vu, K.-P. L., \& Proctor, R. W. (2002). The prevalence effect in twodimensional stimulus-response compatibility is a function of the relative salience of the dimensions. Perception \& Psychophysics, 64, 815-828.

Vu, K.-P. L., Proctor, R. W., \& Pick, D. F. (2000). Vertical versus horizontal spatial compatibility: Right-left prevalence with bimanual responses. Psychological Research, 64, 25-40.

WALLACE, R. J. (1971). S-R compatibility and the idea of a response code. Journal of Experimental Psychology, 88, 354-360.

\section{NOTES}

1. On the basis of the terminology used by Vu and Proctor (2002), we refer to the larger compatibility effect along the dimension of instruction as an advantage. The advantages of the compatibility effect that cannot be solely attributed to the instruction as referred to as prevalence (e.g., the overall compatibility effect when collapsed across the instruction conditions; the larger compatibility effect along an uninstructed dimension relative to the instructed dimension).

2. Rubichi, Nicoletti, and Umiltà (2005) obtained the right-left prevalence effect in a Simon task with hand and foot responses. We discuss this discrepancy in the General Discussion.

(Manuscript received November 3, 2005; revision accepted for publication April 13, 2007.) 